\title{
On the theory of gravitationally-frozen superdense and supermassive objects
}

\begin{abstract}
Zahid Zakir *
Abstract

The black hole paradigm (BHP) is based on an implicit assumption that the collapse occurs quickly not only on proper times of falling particles, but on world time also, and that information about that is retarded only. According to general relativity (GR) in a static field there is a global simultaneity of events, the proper times are slowed down with respect to world time absolutely and these facts are confirmed experimentally by clocks long-term located at different heights. Therefore, the basic assumption of BHP is incompatible with GR, the collapse occurs not quickly, but it needs in really infinity world time. For a falling particle at any finite world time moment its corresponding proper time moment is insufficient for reaching the gravitational radius of the source. As the result, in GR real horizons and physical singularities do not exist, the black holes never be formed. Instead of the black holes GR predicts the gravitationally-frozen states of matter in superdense and supermassive objects. Particles inside of any compact object are frozen by the strong gravitational field so that the time dilation is finite, but maximal at the center and minimal on the surface. In cosmology this fact solves the problem of an initial singularity, in astrophysics allows one to construct a theory of superdense stars, quasars and AGN, in particle physics leads to the ultraviolet finiteness of quantum fields, including quantum gravity.
\end{abstract}

PACS: 95.30.Sf, 97.60.Lf, 98.35.Jk, 98.54.-h, 98.80.-k, 04.60.-m

Key words: black holes, horizon, collapse, singularities, quark stars, quasars, active galactic nuclei, cosmology, quantum gravity

\section{Content}

Introduction

1. The gravitational freezing instead of the collapse .........................................................45

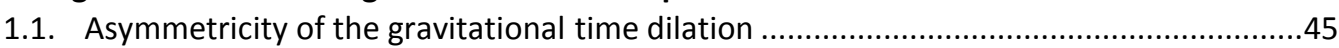

1.2. Criticism of basic assumptions of the black hole paradigm ..........................................47

1.3. The theorems about the lack of horizons and inhomogeneous freezing .........................50

2. Gravitationally-frozen objects in astrophysics ..........................................................52

2.1. Superdense and supermassive stars in phase states of the Standard Model ..................53

2.2. Main properties of superdense and supermassive stars ..........................................53

2.3. Quasars and AGN as inhomogeneously frozen objects................................................54

3. Gravitational freezing in cosmology and particle physics .................................................55

3.1. Big-Bang as refreezing of the supermassive Universe.................................................55

3.2. Freezing of quantum fluctuations and the finiteness of quantum gravity.....................58

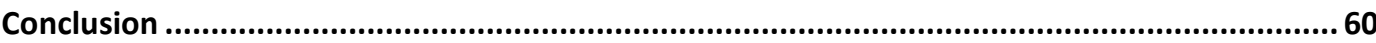

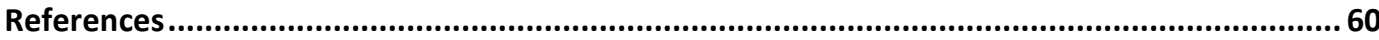

*Centre for Theoretical Physics and Astrophyics, Tashkent, Uzbekistan; zahidzakir@theor-phys.org 


\section{Introduction}

According to general relativity (GR) the processes near a source of static gravitational field should be described not only in terms of proper times $\tau$ of particles freely falling to the source, but also in terms of world time $t$ describing a global simultaneity of events. To any proper time moment $\tau_{1}$ exactly corresponds a definite world time moment $t_{1}$ and both variables are exactly related with each other [1-3]. Here world time approximately counts a mean evolution time of the Universe (with small corrections to a peculiar motion under the cosmic microwave background). Therefore, at consideration of the proper times, slowed down by the gravitational field, we must clearly understand: to what intervals of the expansion time of the Universe correspond these proper time intervals.

However, regardless to this relation, in the black hole paradigm (BHP) [4-10] it has been implicitly supposed, that a falling particle really can reach and even can cross the gravitational radius not only during a finite proper time interval, but a finite world time interval also. There it is supposed that about already happened collapse a distant observer, due to slowing down of light velocity in the field, will be informed later only. Then, starting on such picture, many astrophysical objects have been considered as the formed Black Holes having a real horizon and even a singularity at the center $[6,7]$. It is clear that such point of view of BHP is meaningful only if the local proper times and world time are going synchronously and if the collapse really occurs quickly in world time also. Moreover, in BHP the gravitational radius has an additional meaning of a horizon, a boundary of really existing in space interior region into which the collapsing matter can fall during a finite world time interval.

Therefore, it is necessary to answer to questions, is there in GR such partitioning of space into two regions, what means a horizon in general, and what is a true role of the gravitational radius. Notice, that even the pragmatic researchers, studied the exterior region only, and also the critics of BHP, have supposed the existence of the horizon, and the latter tried to find mechanisms allowing one to prevent the collapse into the horizon. Already Einstein in 1939 [2] attempted to prove impossibility of a collapse in the case of a rotating dust cloud, and he has clearly understood that a falling particle never reaches the horizon and that this should not be a coordinate effect only. Later the alternative models were reduced to models of frozen near a horizon stars with an empty interior [8], or to a stars emitting a supercritical part of their mass [12], and many others where the dynamics of collapse have been complicated by attempting to take into account various hypothetical factors.

In the previous publications [13] I have tried to solve the problems of horizon and singularities by fully excluding from the theory the unobservable interior region by using the physical variables only. But, as it will be shown in the present paper, in fact there is another simple and final solution to the problem. In GR a horizon really never be formed, but interior structure of a real star is related by a new phase state of matter. Thus, the problem of exception from GR hypothetical assumptions contradicting to its basic physical principles has posed correctly, while results 
obtaned at following such methodology are different and a final picture appeared simpler.

As it is known, in GR world time is introduced for joining into a global hypersurface of events simultaneously occurring in the field and it can be measured not only by distant observers, but by local observers also at any point of the field by coordinate clocks going synchronously with distant clocks [5]. Notice, that in the static field world time is most fast going on time since the proper times of all other resting frames of reference are delayed with respect to it at each point due to gravitation, while proper times of freely falling frames are slowed down additionally due to kinematics also. Unlike the kinematical time dilation, which is symmetrical under the observers, the gravitational time dilation is asymmetrical and absolute since both of observers are agree, that the clocks closer to the source are going slower (with exception the kinematical effects).

Therefore, it is necessary to clearly understand that total a world time interval from Big-Bang up to the present time corresponds to such part of proper time intervals of particles falling in strong fields, which even is shorter than an interval needed for the reaching the gravitational radius of the source. As the result, the basic assumptions of BHP are incompatible with GR and in fact there occurs a gravitational freezing of particles inside the compact objects the surfaces of which are close to their gravitational radii.

In this connection, in the present paper it is shown that at a correct definition of the relation between the proper and world times of events, GR leads to a new theory of superdense and supermassive states of matter. According GR, in terms of world time, particles inside of any compact object will be frozen by the strong gravitational field beginning from the center, where proper time dilation is maximal, up to the surface where it is less. Still Feynman noted that the center of the Earth per day or two is younger than its surface [11]. As it will be shown in the paper, any other object in principle does not differ from the Earth; a difference will be in a degree of freezing only.

In the Part 1 of the paper it is discussed absolute character of the time dilation in the gravitational field with respect to world time, it is shown the incompatibility of basic assumptions of BHP with GR, and also the theorems about the lack of a horizon and physical singularities are proven. In the Part 2 main properties of the gravitationally-frozen objects in astrophysics are briefly described. In the Part 3 a role of gravitational freezing in cosmology and in quantum gravity is considered. In the forthcoming publications more detailed consideration of the proposed approach will be presented. 


\section{The gravitational freezing instead of the collapse}

\subsection{Asymmetricity of the gravitational time dilation}

Einstein's equations on outer region of a centrally-symmetric source lead to Schwarzschild's linear element written in terms of curvature coordinates [3]:

$$
d s^{2}=\left(1-r_{g} / r\right) c^{2} d t^{2}-\frac{d r^{2}}{1-r_{g} / r}-r^{2} d^{2} \Omega,
$$

where $r_{g}=2 G M / c^{2}$ is the gravitational radius and there are formally two singularities of the metric.

The first singularity at the center $r=0$ there is in the Newtonian potential also, but for point sources only. In GR it becomes physical only if object's radius can be less than $r_{g}$, and in BHP this has been postulated additionally. How objects GR really predicts, will be considered further in section 1.3.

Another singularity on the gravitational radius $r=r_{g}$ in BHP has been considered as an artificial one, disappearing at the transition from "unsuccessful" curvature coordinates $t, r$ to more convenient ones, particularly to the local coordinates of freely falling particles [4-9]. In connection with such statements, and also due to the general covariance of the equations, we will clearly understand what of properties of solutions of field equations have a real physical meaning at the studying of physical properties of objects, and what are misleading coordinate effects only.

Firstly, at neighborhood of each point of a static field it is possible to introduce a set of coordinate clocks measuring the world time $t$. They are constructed going faster than local standard clocks so, that always to be synchronous with distant observer's clocks [5]. In other words, on a hypersurface of simultaneity of the distant observer all coordinate clocks will show the same moment of world time. Therefore just on the basis of such set of clocks, i.e. on world time, it is possible to formulate correctly the Hamiltonian dynamics in this field. Of course, the coordinates of the distant observer can be subjected to the Poincaré group transformations, but only in the rest frame of the source we deal with pure gravitational effects, while all other moving frames introduce kinematical complications only.

In GR, than a point is closer to the source, than the proper times are showered stronger and the coordinate clocks go faster with respect to a standard clocks at this point. For this reason around the spherical source a proper time interval $\Delta \tau$, measured by a rested standard clock, is related by a world time interval $\Delta t$, measured by a coordinate clock rested at the same point as:

$$
\Delta \tau=\Delta t \sqrt{1-r_{g} / r} \text {. }
$$

Secondly, here it is important for us the fact that for the comparison of the proper time interval $\Delta \tau$ with a world time interval $\Delta t$ one should not need in signaling to the distant observer. It is enough to compare the indications of the neighboring standard and coordinate clocks. 
And thirdly, a ratio of these time intervals is invariant since they are measured by relatively resting standard and coordinate clocks near the same point. Observers on any reference frame will obtain the same relation (2) for the time intervals measured at the given point by these two neighbor clocks.

In the Newtonian theory time is absolute and the proper times are identical with world time. There it has been supposed that a photon emitted in a strong field will have the same frequency (measured in world time), as a photon emitted by a distant source and that the frequency shift occurs only during the propagation from an emitter to a receiver. It is supposed that the redshift occurs due to loss of photon's "kinetic" energy (identified by $h v$ ) at escaping the gravitational field, and the violetshift - because of increasing of that "kinetic" energy at falling in the field (the role of an "effective mass" plays $\left.m=h v / c^{2}\right)$ :

$$
h v-\frac{G M}{r}\left(\frac{h v}{c^{2}}\right)=h v_{0}-\frac{G M}{r_{0}}\left(\frac{h v_{0}}{c^{2}}\right) .
$$

Thus, in the Newtonian gravity the gravitational frequency shift of photons from a source in the gravitational field and registered at different heights is given by:

$$
\frac{v-v_{0}}{v_{0}} \approx \frac{r_{g}}{2 h v_{0}}\left(\frac{1}{r}-\frac{1}{r_{0}}\right) \text {. }
$$

Formally GR gives in the first order the same formula, but due to the time dilation in the gravitational field the light source emits the frequency shifted photon, further during propagation the frequency of which (measured on world time) does not change (see [17]).

Since in these two treatments of gravitation a physical nature of the frequency shift of the registered photon is explained differently, additional effects have been studied with corresponding experiments which have allowed one to confirm the GR treatment and to reject the Newtonian (see [14-17]). In the Newtonian theory the indications of clocks are independent on their duration time at different heights. In GR the standard clocks in the gravitational field are going slowly and the total difference $\Delta \tau$ of indications of two clocks will be proportional to world time duration $t$ of placing a clock in the field at difference of heights $\Delta h$ :

$$
\Delta \tau \approx t \cdot g \Delta h / c^{2}
$$

where $\mathrm{g}$ is the gravitational acceleration.

The experiments $[15,16]$ have confirmed the GR formula (5) that $\Delta \tau$ is proportional to the duration time $t$ of the clocks at different heights, and now this effect is taken into account in the satellite systems of global positioning [14]. Thus, the fact of slowering of proper times in the gravitational fields with respect to the distant observer's time is confirmed experimentally and is using practically. 


\subsection{Criticism of basic assumptions of the black hole paradigm}

On the basis of a formally mathematical approach to spacetime geometry in strong gravitational fields of compact sources it has been developed BHP [4-10] which was a standard paradigm within last four decades. The theoretical part of the paradigm contains a hypothesis about a fast collapse and formation of black holes, and also the hypotheses about processes inside the horizon [5-7,9], including theorems about inevitability of a physical singularity at the center $[6,7]$. The observational part of the paradigm is reduced to attempts to find out evidences of the horizon on candidates to black holes as which supporters of BHP consider all stars densely than neutron stars, and also the central parts of quasars and most of galaxies [10].

However, BHP is based mainly on few basic implicit assumptions which although have not been clearly formulated in publications, but nevertheless, they have been in view when founders and followers of the paradigm have predicted the existence of physical objects with real horizons.

In 1939 Oppenheimer and Snyder in the paper [4], established BHP, wrote: «...Since the pressure of the stellar matter is insufficient ...the star will contract, and its boundary $r_{b}$ will necessarily approach the gravitational radius $r_{0}$...The star thus tends to close itself off from any communication with a distant observer... From the point of view of a distant observer, an infinite time for this asymptotic isolation to be established, for an observer comoving with the stellar matter this time is finite and may be quite short. ... As $t$ tends toward infinity, $\tau$ tends to a finite limit $\left(\tau_{0}\right)$. After this time $\tau_{0}$ an observer comoving matter would not be able to send a light signal from the star... For a star which has a mass $10^{33} \mathrm{~g}$ this time $\tau_{0}$ is about a day...»

As we see, by "after this time $\tau_{0}$ " authors understand not after the infinite world time $t>\infty$, which is physically meaningless, but they have implicitly supposed the same order as $\tau_{0}$ a finite world time interval, i.e. also «about a day». The same logical jump has been repeated practically in all subsequent texts about BHP. As an example we present the treatment of the relation of times in the well-known book [5]:

«...Nor can it reach a static equilibrium state, since there exists no such state for so large a mass. There remains only one alternative; the supercritical mass must collapse through its "gravitational radius" $r=2 M$, leaving behind a gravitating "black hole" in space. (p. 819)

...The gravitational radius is a perfectly well-behaved, nonsingular region of spacetime, and nothing there can prevent the explorer from falling on inward.

By contrast, deep inside the gravitational radius, at $r=0$, the traveler must encounter infinite tidal forces, independently of the route he uses to reach there. (p. 822)

...One therefore suspects that all the physics at $r=2 M$ is consigned to $t \rightarrow \pm \infty$ by reason of some unhappiness in the choice of the Schwarzschild coordinates. Better coordinate system, one begins to believe, will take these two "points at infinity". (p. 824) 
No matter how stiff may be the matter of which a (spherical) star is made, once its surface has collapsed within the gravitational radius, the star will continue to collapse until its surface gets crushed in the singularity at $r=0$. (p. 846)

...To the distant astronomer, the collapsing star appears to slow down as it approaches its gravitational radius: light from the star becomes more and more red-shifted. Clocks on the star appear to run more and more slowly. (p. 846)

...For all practical purposes the phrase "black hole" is an excellent description. The alternative phrases "frozen star" and "collapsed star"... emphasize an optical-illusion aspect of the phenomenon. (p. 874)»

As we see, here it is supposed that for the distant observer a hole in space will be formed during the same order time interval, as for the local observer near a star. An observed large world time interval of the collapse for the distant observer is treated as "an optical illusion», caused by dilation of a propagation time of light up to the observer, whereas the real collapse has occurred quickly when luminosity quickly decreased.

Thus, the first basic implicit assumption of BHP is that, as well as in the Newtonian theory, the proper times of distant and falling observers have been supposed as going synchronously. As the result, it was meant, that at a collapse a particle cross a horizon at short temporal intervals, both on proper time, and on world time. The distant observer simply "cannot observe" this already occurred collapse because of the late delivering of an information about that due to slower propagating light signals. Thus, a difference of GR from the Newtonian gravity has been reduced in this case mainly to the difficulties with the late informing of the distant observers.

However, as it has been considered in Section 1.1, the experiments showing the irreversible slowing down of proper times in the gravitational field, disprove this assumption of BHP when the collapse is supposed as occurring during a finite world time. If the proper times of the freely falling observers are really going slower with respect to world time, about which the local observers can know by means of neighboring coordinate clocks, then the falling particles approach $r_{g}$ at really infinite world time. Therefore, at any fixed moment of world time the freely falling observers had not time to reach $r_{g}$ in proper time also, since their proper times become practically frozen in terms of world time.

The second implicit assumption of BHP is that after the transition to local systems of reference of free falling particles, it is supposed possibility to construct a global temporal axis from the local intervals of their proper times. Then, spacetime diagrams and trajectories in terms of such global proper time coordinate have been drawn. But it is admissible in the Newtonian gravity only.

In GR, however, the intervals of physical coordinates measured by the local standard rods and clocks, are directly unintegrable. The local physical frames at different points of the field are subjected to the local Lorentz rotations due to gravitation and a relative motion. As the result, their standard clocks cannot be globally synchronized, and a hypersurface of simultaneity exists at one a moment only. We must to take into account the changing of length of standard rods and rate of standard clocks at different points of field and in moving local frames. This can be 
done by expressing the length of standard rods and proper time intervals in the field through the well-defined global physical coordinates.

In a static field simplest of such global physical coordinates are the curvature coordinates with the Schwarzschild metrics. At the same time, all other coordinate systems are or various parameterizations of the curvature coordinates and have not independent physical meaning, or incorrectly constructed and unphysical ones due to ignoring unintegrability of the local physical coordinates.

As an example of the artificial singularity usually is given singularity on a pole of a spherical coordinates at $\theta=0$ when corresponding component of metrics vanishes: $g_{\varphi \varphi}=r^{2} \sin ^{2} \theta=0$. Then it has been concluded that the Schwarzschild singularity is the same kind coordinate effect only [5]. In fact, this example shows the physical character of the radial and temporal curvature coordinates. Really, at approaching the pole thicknesses of the objects do not decrease along parallels with respect to their thickness at equator, and, therefore, here the polar singularity is obviously coordinate effect only, unrelated with the physical size of objects. However, at approaching of a gravitational radius a radial size of bodies are really contract with respect to their size at large distance, and the standard clocks are really going slowly than distant clocks. Therefore the Schwarzschild metric exactly expresses a pure influence of gravitational field to the physical properties of objects. On the contrary, all other, using in BHP, artificial global coordinate systems introduce kinematical and geometrical complications in addition to gravity's influence to properties of bodies and events.

The third basic assumption of BHP consists in the postulate about the real existence of sources, the radii of which are less than their gravitational radii and around which there is a real horizon.

For the reaching the horizon by a sample particle, this horizon must be existed before that. For its formation, the particles of a source should cross the horizon. But, this means that the particles of the source should crossed the horizon at a finite world time moment which is impossible in GR.

In addition, since the first publications on BHP, the most of attempts to construct the models of collapse, even in case of a dust matter, were reduced to the introduction of the non-stationary, but globally defined frames of reference. The problems with construction of such global non-uniform frames of reference are considered above. In BHP as a global temporary coordinate it has been used usually the proper times of freely falling particles which in GR is unintegrable as measured by the local standard clocks going by different rates at different points and at different moments.

To build a global time axis, according to GR, it is necessary to express all local proper time intervals across a globally defined, homogeneous (having identical units everywhere) an axis of the world time measured in any point of a field by synchronously going coordinate clocks. But, then we again conclude, that any particle of the collapsing matter cannot reach $r_{g}$ at a finite world time interval, and the considered assumption of BHP in fact is incompatible with GR. 
At last, the fourth assumption of BHP, residual from pre QCD epoch, consists in that a cold degenerate neutron fluid has been supposed as a last stable state of matter and that other more dense stable states of stars, preventing further squeezing, do not exist.

Already there are huge publications about quark stars, boson stars, etc., and it is obvious that one must take into account all possible phase states of matter allowed by the Standard Model. Nevertheless, in most of literature about BHP till now it is repeated the initial statement of founders of BHP about that neutron star is last stable state after excessing the critical mass of which the collapse is inevitably. The question on what kind of phase states of matter after neutron stars GR really predicts, we shall discuss in the Part 2 of the paper.

\subsection{The theorems about the lack of horizons and inhomogeneous freezing}

At free falling of a sample particle, at any finite moment of world time $t$ it is located at $r(t)>r_{g}$. Below it will be shown that to this value of the radial coordinate corresponds a proper time moment $\tau[r(t)]$ which is always less than a moment $\tau\left[r_{g}(\infty)\right]$ when it would be reach $r_{g}$, i.e. $\tau[r(t)]<\tau\left[r_{g}(\infty)\right]$.

Let's consider an example of a thin spherical dust shell, the particles of which freely fall to the center of symmetry. The gravitational radius $r_{g}=2 G M$ is defined by a total energy of the sphere at very large radius. The metric outside and on the shell $r \geq R$ is the Schwarzschild metrics (1), while the interior metric of the shell at $r<R$ is constant, which for $g_{00}(r)$, due to the continuity, should be chosen as equal to the metric on the surface:

$$
g_{00}(r)=1-\frac{r_{g}}{R}, \quad r \leq R .
$$

According to GR, small intervals of proper time are related strongly by the world time intervals according to (2). If particles fall from rest at spatial infinity, then at any $r$ they will have a coordinate velocity:

$$
v(r)=\frac{d r}{d t}=-\sqrt{\frac{r_{g}}{r}}\left(1-\frac{r_{g}}{r}\right) .
$$

Let this dust sphere had necessary coordinate velocity $v_{1}\left(r_{1}\right)$ at any large, but finite radius $r_{1}$ at initial moments of world time $t_{1}=0$ and proper time $\tau_{1}=0$. Then, a world time moment $t$ of falling dust shell up to the radius $r<r_{1}$ is defined by the equations of motion as:

$$
t=\frac{1}{r_{g}^{1 / 2}} \int_{r}^{r_{1}} d r \frac{r^{3 / 2}}{r-r_{g}}=C\left(r_{1}\right)-\frac{2 r^{3 / 2}}{3 r_{g}^{1 / 2}}-2\left(r_{g} r\right)^{1 / 2}+r_{g} \ln \left|\frac{r^{1 / 2}+r_{g}^{1 / 2}}{r^{1 / 2}-r_{g}^{1 / 2}}\right|,
$$

where $C\left(r_{1}\right)=$ const. This relation gives an exact dependence $r(t)$ of the areal radius of the dust shell on the world time $t$. As we see, due to the presence of the 
logarithmic term, at any finite world time moment $0<t<\infty$, the dust shell is located outside of own gravitational radius: $r(t)>r_{g}$.

Let's consider a corresponding proper time $\tau[r(t)]$ of the particles of the shell at the same areal radius $r(t)$ :

$$
\tau[r(t)]=\frac{2}{3 r_{g}^{1 / 2}}\left[r_{1}^{3 / 2}-r^{3 / 2}(t)\right] .
$$

The proper time moment $\tau_{g}$, when the shell would approach $r_{g}$ at $t \rightarrow \infty$, formally is equal to:

$$
\tau_{g}=\frac{2}{3 r_{g}^{1 / 2}}\left(r_{1}^{3 / 2}-r_{g}^{3 / 2}\right)
$$

Thus, at any finite world time moment $t<\infty$, when $r(t)>r_{g}$, the corresponding proper time moment $\tau[r(t)]$ remains less than $\tau_{g}$ :

$$
\tau_{g}-\tau(t)=\frac{2}{3 r_{g}^{1 / 2}}\left[r^{3 / 2}(t)-r_{g}^{3 / 2}\right]>0, \quad r(t)>r_{g} .
$$

Thus, at any finite world time moment $0<t<\infty$, and corresponding proper time moment $0<\tau[r(t)]<\tau_{g}$, the dust shell does not reach own gravitational radius. The moment of proper time $\tau_{g}$ never will come to since the infinite world time $t \rightarrow \infty$ never will be expired. The events after the infinity world time of the Universe, obviously, are the events which never occur.

If the shell cannot reach $r_{g}$, then inside of it at any finite world time moment an event horizon also does not arise. The temporary and radial components of the metric remain regular both outside and inside the dust shell.

The same result will be obtained for any layer of a centrally symmetric dust ball of finite mass, the particles of which fall radially. Any layer does not form a horizon neither outside, nor inside the layer, while all other layers change a time dilation coefficient $g_{00}^{-1}\left(R_{n}\right)$ of other layers to a finite value.

The increasing of the time dilation coefficient at the surface due to interior layers will be less than the same at the center $g_{00}^{-1}(0)$. Really, any higher layer contribute to the time dilation at the center constantly and exactly as on the layer, while the contributions of interior layers to the time dilation on the surface will be decreased due to the distance.

Thus, as we see on the example of the exactly solvable model of a dust cloud, as well as a dust ball, the qualitatively new properties of objects in strong gravitational fields obey some general restrictions, main of which we formulate in the form of following theorems: 
Theorem 1 (about impossibility to reach $r_{g}$ for outer particles and surface):

If in a static centrally-symmetric gravitational field of a compact source a sample particle, falling to the source from outside or on the surface beginning at zero moments of world and proper time $t_{0}=\tau_{0}=0$, at any finite moment of world time $0<t<\infty$ has such moment of proper time $\tau[r(t)]$ which will less than a moment of proper time $\tau_{g}$ when the particle would be reach the gravitational radius: $0<\tau[r(t)]<\tau_{g}$.

\section{Theorem 2 (about the lack of a horizon and singularities):}

Since at any finite moment of proper time $\tau[r(t)]<\infty$ of the local observers, corresponding to a finite moment of world time $0<t<\infty$, the surface radius $R$ of a compact object exceeds its gravitational radius: $R>r_{g}$ , then at any spatial point the spacetime interval is time-like along worldlines of massive particles: $d s^{2}>0, r \geq 0$, the metric is regular: $g_{00}(r)>0$, $0<\left|g_{11}(r)\right|<\infty$ and a horizon and a singularity do not arise.

\section{Theorem 3 (about inhomogeneous gravitational freezing):}

Inside of a compact object at any finite world time moment the gravitational time dilation coefficient is finite, and it is maximal at the center and minimal at the surface of the object: $g_{00}^{-1}(R) \leq g_{00}^{-1}(0)<\infty$.

Proofs of these theorems in general case are similar to the case of centrally symmetric dust cloud at correct taking into account of the relations between the proper and world times. The formal proofs will be considered in forthcoming publications.

In the case of rotating objects the role of a gravitational radius will play an external radius of freezing defined by zero of the temporary component of the metric. For the charged sources the gravitational radius will be defined, firstly, by a pure mass of object without the contribution of its electric field, and secondly, by the contribution of the electric field's energy.

\section{Gravitationally-frozen objects in astrophysics}

Thus, GR excludes the formation of the black holes with the real horizons and physical singularities at the center. This clear and unambiguous conclusion of GR is very important for astrophysics, cosmology and quantum theory since now unreasonable restrictions of fundamental physics disappear and instead the new perspectives for the modeling of the real structure of superdense and supermassive objects are opened.

The questions about matter's phase states which will be appeared in these cases, requirements and conditions for their formation and transformations, etc. now 
become quite physical problems with realistic solutions and observable predictions instead of former pure mathematical and fantastic scenario of BHP.

\subsection{Superdense and supermassive stars in phase states of Standard Model}

The superdense and supermassive objects should be considered, first of all, as a matter in various phase states allowed by the Standard Model. Real stars have complicated internal structure and there is no even a pure neutron star. Therefore, even at excess of a critical mass of neutron stars, there may be formed the structures containing a mixture of various fermions and bosons, including the quark and boson matter.

In addition to the models of cold stars, also it is necessary to study the models of hot quark stars, since the asymptotic freedom and pointness of quarks lead to various strongly squeezed hot states with high pressure.

Now it also becomes actual studying of boson stars and models of compact objects containing hypothetical particles, for example, subquarks, especially, formed at early periods of the expansion of the Universe.

Unlike usual stars, in the case of relativistic stars an essential role will probably play the electrical charge of the stars appearing because of escaping of a part of electrons by powerful radiation flows from the lower to upper layers and to the atmosphere. The rotation of such charged star before freezing will create such currents on its surface, in the atmosphere and on a disk, and also so powerful magnetic fields, that many unusual properties of these objects, possibly, may be related by these reasons.

\subsection{Main properties of superdense and supermassive stars}

Let us consider the states of matter in the strong gravitational fields using the general properties of the frozen objects formulated in section 1.3.

Main new property of such relativistic stars (including quasars and nuclei of galaxies), except lack of a horizon, is that now we deal with a new phase state of matter which is gravitationally-frozen on world time, but which in other side, is very hot on proper time. Thus, such objects will represent more likely not "cold", but "hot" stars in the sense of local temperatures. Since the local temperatures and pressures will be comparable with rest masses of particles, the equations of state and equilibrium conditions of such ultra-relativistic states will essentially differ from simple models of a cold fermionic gas.

Being similar to the frozen ultra-relativistic plasma, such objects become the powerful sources of the continuous radiation, especially, in radio region (because of redshifting of all frequencies), are partially charged because of escaping from surface a part of electrons by radiation flows, and have powerful magnetic fields because of rotation.

At attempts to find out horizons on candidates for black holes, it was revealed that some of such objects are more silent than neutron stars and that the thermonuclear explosions at falling of massive objects on their surface do not observed. The compact supermassive objects at the center of many galaxies are relatively silent also. 
These facts do not contradict to the new paradigm of the gravitationallyfrozen objects, and moreover, probably, may confirm this picture. Really, unlike a surface of a neutron star, where redshift is insufficient, on the surface of the gravitationally-frozen objects the time dilation coefficient is very large. Therefore, the notion "explosion" on local time has other sense on world time since such locally fast phenomena will be observed by the distant observers as a slow changing of properties during longer time. Such changing of properties in the form of «delayed explosions», continuing hours and days are really observed from time to time practically at all of such objects. Although the mechanisms of these phenomena may be complicated, but it is clear, that the required dilated explosions also probably contribute to the total effect. Moreover, such dilated explosions may be used further for the defining a time dilation coefficient near the surface of such compact frozen stars.

One of the characteristic features of stars in the Galaxy with the gravitationally-frozen surface will be the gravitational redshift of atmospheric spectral lines which not have, unlike extragalactic quasars, the cosmological nature. Probable, a part of the objects catalogued as quasars may be such stars in the Milky Way. They may be distinguished, firstly, by traditional methods, i.e. by growing of their density in the Galactic equator and by measuring of their parallax. Secondly, the part of them can possess too high luminosity in all wavelengths at the given redshift, and also their parameters can vary too quickly, etc. In any case observers should understand, that GR predicts two essentially different types of objects having redshifts and further should be paid special attention to their differentiating.

New opportunities will be opened by GR in the construction of the scenario for the supernovae explosions and for the synthesis of chemical elements. So, the synthesis of elements not only can occur during very short instants of contracting and explosion of the supernovae. They can be formed during long time in the gravitationally-frozen superdense stars and to be escaped out at the achievement of an instability point under the rapid refreezing, i.e. at the explosion of such special type of supernovae.

Notice, that the theories of accretion of matter and formation of disks and jets near compact relativistic objects will not change sufficiently, except for those their parts which have based on the horizon hypothesis. Moreover, they will be developed and simplified since now there is no need to try to explain all observed properties of the compact objects by disks and jets, and there a part of them can explained by the surface effects that will allow one to create a realistic picture of processes around such objects.

\subsection{Quasars and AGN as inhomogeneously frozen objects}

The relativistic objects of stellar masses can be studied by means of models which are close to the existing ones, with addition of new structures, interactions, the Standard Model phase states and gravitational freezing. In the case of supermassive relativistic objects, such as extragalactic quasars and AGNs, there appear essentially new properties. 
Firstly, the redshifts of lines of compact extragalactic objects, except of cosmological redshift, contain also the contribution of the gravitational redshift, and new methods allowing one to define the contribution each of mechanisms should be found.

Secondly, the gravitationally-frozen matter will be superdense only in compact central parts of quasars and AGNs. In the closer to surface layers the matter density may be ordinary, although will be highly frozen on world time. Therefore, such objects will have a complicated interior structure with a matter in various phase states.

Thirdly, now one can explain, in the framework of GR and Standard Model mechanisms, the super-luminal radiation of quasars, AGN and GRB without additional hypotheses. Except for the known mechanisms based on an accretion and existence of disks and jets, there appear the interior and surface radiations. Any stationary or quasi-stationary state of the supermassive objects will be accompanied by the powerful radiation flows.

At last, in the fourth, the theory of the origin of quasars and the galaxies, explaining also their possible genetic relations, now can be developed on a new fundamental basis. Particularly, if up to now BHP prohibited the explosion of a supermassive black hole, now the explosion of the supermassive frozen object will be possible if there occurs a phase transition at the synthesis of new particles and nuclei at which enough energy for refreezing by explosion can be liberated.

\section{Gravitational freezing in cosmology and particle physics}

\subsection{Big-Bang as refreezing of the supermassive Universe}

In simplest cosmological models, based on GR and the cosmological principle, the proper times $\tau$ of clocks comoving to matter have been used as a global temporal coordinate. As the result, there appeared the cosmological singularity in the form of infinitely dense state at initial moment $\tau=0$. Here, as well as in a static field, in fact the problem has been created artificially.

Firstly, in GR the description of the extended on time processes directly in terms of proper times requires to be careful if the rate of the proper times varies during the evolution. At different points A and B are defined "A-time" and "B-time" and there it is possible to compare local intervals of the proper times of these two points only if a common "time" is defined. For this purpose the local proper time intervals should be projected on a homogeneous axis of a "global cosmological time", as which it can be chosen the proper time $t$ of a state when the Universe will be expanded up to very large size and there will be no gravitational and kinematical time dilations.

This cosmological coordinate time goes by a constant rate during full evolution time of the Universe. The proper times of all other observers will be showered with respect to it, and at the expansion they will approach to its rate. Then the proper time intervals will be expressed at any fixed spatial point as (up to kinematical corrections):

$$
d \tau=g_{00}^{1 / 2}(R) d t
$$


where $R$ is the curvature radius. This homogeneous temporary coordinate (world time) $t$ is measured at each spatial point by the coordinate clocks going faster than the standard ones according to (12).

Secondly, most of former cosmological models were based on the additional assumption that 3-dimensional space of a constant curvature can exist without embedding into a higher dimensional space. If we do not consider unreal cases of the universes with infinity mass, i.e. with a finite density and infinity volume, and to be restricted to the most plausible closed Universes, the above-mentioned assumption about the 3D space become unreasonable.

Really, in geometry there are obvious facts that the 1D sphere - a circle exists only at embedding even into a 2D surface, and 2D sphere - at embedding into $3 \mathrm{D}$ space. In both cases a D-dimensional sphere divides a (D+1)-dimensional space on two parts - interior and exterior regions. Thus, D-dimensional "area" $S$ of the sphere is related by $(D+1)$-dimensional "volume" $V$ of the interior region as:

$$
S_{(D)}=b_{(D)} V_{(D+1)}^{q}, \quad q=\frac{D-1}{D+1},
$$

where $b_{(D)}$ - is a constant.

For example, the length $S_{(1)}=2 \pi r$ of a circle on a plane is proportional to square root of area of this circle: $S_{(1)}=b_{(1)} V_{(2)}^{1 / 2}$, where $V_{(2)}=\pi r^{2}$ and $b_{(1)}=2 \pi^{1 / 2}$. The area $S_{(2)}=4 \pi r^{2}$ of 2 -sphere in $3 d$ space is proportional to the ball's volume $V_{(3)}=4 \pi r^{3} / 3$ in the degree 2/3: $S_{(2)}=b_{(2)} V_{(3)}^{2 / 3}$, where $b_{(2)}=3^{2 / 3}(4 \pi)^{1 / 3}$.

Further, prolonging on an induction, we obtain that the 3-sphere's 3D volume $S_{(3)}=2 \pi^{2} r^{3}$ encloses 4D interior volume $V_{(4)}=\pi^{2} r^{4} / 2$ and we have $S_{(3)}=b_{(3)} V_{(4)}^{3 / 4}$, where $b_{(3)}=2^{7 / 4} \pi^{3 / 2}$. Therefore, if the interior 4-volume $V_{(4)}=0$ vanishes, then corresponding 3-volume of 3-sphere vanishes also, i.e. 3-sphere exists only at embedding at least into 4-space.

Thus, we shall consider further, that the Universe is a 3 -sphere embedded into 4D space, and its evolution is described in terms of the global cosmological time $t$. The observational effects and data, which can confirm these model assumptions, will be discussed in forthcoming papers.

The solutions of Einstein's equations for the metrics of a thin 3-spherical dust shell in a 4-space are known and give the linear element:

$$
d s^{2}=\left(1-a^{2} / R^{2}\right) c^{2} d t^{2}-\frac{d R^{2}}{1-a^{2} / R^{2}}-R^{2} d^{3} \Omega,
$$

where $a$ - is the gravitational radius of the Universe depending on its mass. Expansion of the Universe is a free outfalling of this 3-sphere. For the dependence of proper times on $t$ we have (up to kinematical factors):

$$
d \tau=d t \sqrt{1-a^{2} / R^{2}} .
$$


Let's consider a simple case of the Universe with a critical energy $M_{0} c^{2}$ where the expansion will stop only at infinity radius. Then, the conservation of energy gives:

$$
M_{0} c^{2}=M_{0} c^{2} \frac{\sqrt{1-a^{2} / R^{2}}}{\sqrt{1-v^{2} / c^{2}}},
$$

which leads to:

$$
v=\frac{d R}{d \tau} \frac{1}{\sqrt{1-a^{2} / R^{2}}}=\frac{d R}{d t} \frac{1}{1-a^{2} / R^{2}}=\frac{a}{R} .
$$

These equations give us the dependences of proper and coordinate times on the curvature radius:

$$
\begin{gathered}
\tau=\frac{1}{2 a} R \sqrt{R^{2}-a^{2}}+\frac{a}{2} \ln \left|R+\sqrt{R^{2}-a^{2}}\right|+\text { const }, \\
t=\frac{1}{2 a} R^{2}+\frac{a}{2} \ln \left|R^{2}-a^{2}\right|+\text { const } .
\end{gathered}
$$

Let $\tau_{0}$ is the present moment, and $\tau_{a}$ - is a moment of proper time, corresponding to approaching the gravitational radius $R_{a}=a$. Then, to this finite proper time interval $\tau_{a}$ corresponds infinity coordinate time $t_{a} \rightarrow-\infty$ :

$$
\begin{gathered}
\tau_{a}-\tau_{0}=-\frac{R_{0}}{2 a} \sqrt{R_{0}^{2}-a^{2}}+\frac{a}{2} \ln \left(\frac{a / R_{0}}{1+\sqrt{1-a^{2} / R_{0}^{2}}}\right), \\
t_{a+\varepsilon}-t_{0}=\frac{1}{2 a}\left(a^{2}-R_{0}^{2}+a^{2} \ln |0+\varepsilon|\right) \rightarrow-\infty .
\end{gathered}
$$

In fact, total energy of the Universe, most likely, less than the critical energy $M_{0} c^{2}$ and the expansion will stop on a value of radius $R_{m}$. After that will begins a new free falling up to new frozen state. Since at each moment of global time $t(R)<\infty$ one has $R>a$, and then $\tau<\tau_{a}$, where $\tau_{a}$ is the future proper time moment corresponding to reaching of the gravitational radius.

Thus, at the beginning of expansion the Universe, apparently, was in a partially frozen state in terms of world time, although, in terms of the proper time it was very hot. Therefore, the Big-Bang and the expansion represent in fact the refreezing of the Universe.

Thus, as in a static field, here the horizon and singularities do not arise. The gravitational radius defines a size of region, at the maximal approaching of which the time dilation in the Universe reaches maximally admissible by the equations of state a value, and then the expansion begins as fall off.

The important consequence of it is that the estimations of an age of the Universe should be improved since the gravitational time dilation leads, especially in early periods, to the large differences of proper times from the global time. Therefore, total age of the Universe in terms of the present time scale will be different than the 
proper age of matter due to the cosmological time dilation. Thus, the age of the Universe in time units of our epoch will essentially be increased at the same proper age of matter if to take into account the strong time dilation in the dense states.

Thus, in simplest models of the relativistic cosmology the cosmological singularity before Big-Bang disappears without additional hypotheses and further BigBang can be considered as fall off the Universe from the gravitationally-frozen state due to the internal phase transitions.

\subsection{Freezing of quantum fluctuations and the finiteness of quantum gravity}

Gravitation is only one from the known interactions, the theory of which is non-renormalizable. This means that if in quantum gravity one calculates the graviton loop diagrams on a flat background spacetime, the divergences cannot be removed completely by a finite number of counterterms.

Below it will be shown that here the problems with infinities also have been created artificially and that they disappear at more accurately taking into account the requirements of basic principles of the theory. Particularly, the assumption about the flat background spacetime was incorrect since it is necessary to take into account the external gravitational field creating at any moment of world time by the total energy $E=E_{\mathbf{p}}+E_{\mathbf{p}^{\prime}}+\ldots$, of the particles in the loop diagram, where $E_{\mathbf{p}}=\sqrt{\mathbf{p}^{2}+m^{2}}$.

As the Coulomb field, the external gravitational field of particles is not quantized and it can be described at any moment of world time through the Schwarzschild metric (in the "rest frame" where the total 3-momentum of the loop's particles vanishes and as a first approximation). Then the temporary part of the metric leads to the gravitational redshift of frequencies of particles in the loops. Therefore, when wavelengths of particles of the loop are comparable with a gravitational radius corresponding to an instantaneous total energy of the loop, the frequencies of particles become highly redshifted and effectively cutted.

Let us consider the processes described by high-frequency parts of loop diagrams near the Planck energy $E_{p l}=\sqrt{h c^{5} / G}$, when the particles are localized at the Planck distances $l_{p l}$ (we restore units $c \neq 1$ ). But particles with the energy $E_{p l}$ have a gravitational radius which also is practically equal to $l_{p l}$. Really, it is known that if the quantum mechanical wavelength of a particle is equal to its gravitational radius, we have:

$$
\frac{2 G E_{p l}}{c^{4}}=\frac{h c}{E_{p l}},
$$

and the gravitational radius also is of order $l_{p l}$ :

$$
r_{g}=\frac{2 G E_{p l}}{c^{4}}=\sqrt{\frac{2 G h}{c^{3}}}=\sqrt{2} l_{p l} \text {. }
$$

Thus, the system of particles in the loop should be considered as a source, around of which there is the Schwarzschild metric on its rest frame at any moment of 
world time (in the spherical approximation). There as less the localization area, then fluctuations of energy are higher and the gravitational field of the system of particles is stronger, and then the redshift of frequencies of the same particles is higher.

Really, the localization region of quanta in the loop is of order of their wavelengths which are inverse proportional to the coordinate frequency $v$ (measured on world time): $\lambda=c / v \approx r$. The gravitational radius of the loop is proportional to the total energy which is proportional to the coordinate frequency $v$. As the result, we have:

$$
v=v_{0} \sqrt{1-\frac{r_{g}}{r}} \approx v_{0} \sqrt{1-\frac{2 G h v}{c^{4}} \frac{v}{c}}=v_{0} \sqrt{1-\frac{2 v^{2}}{v_{p l}^{2}}},
$$

where $v_{p l}=\sqrt{c^{5} / G h}$ - is the Planck frequency. Therefore, we find the following relation between the proper frequencies of quanta $v_{0}$ and their coordinate frequencies $v$ :

$$
v \approx \frac{v_{0}}{\sqrt{1+2 v_{0}^{2} / v_{p l}^{2}}},
$$

As we see, at arbitrary high proper frequencies $\nu_{0} \gg \nu_{p l}$, the observable coordinate frequencies will not exceed the Planck frequency: $v<v_{p l}$.

Therefore, the static self-gravitational field of the loop plays the role of a regularization factor for the high-frequency contributions, restricting their frequency for the exterior observer by $v_{p l}$. Then, the high proper frequency quanta of any field in fact become frozen near $l_{p l}$.

As the result, the loop diagrams of quantum gravity become finite due to the freezing of the high frequency gravitons at $l_{p l}$ where there is the self-regularization of the loop. Therefore, at the renormalization in the perturbation theory of quantum gravity it is enough to take the physical observables as sums of finite bare values and finite quantum corrections so that the measurable values coincide with the experiment.

In quantum gravity there small not only logarithmic, but all contributions also because of weakness of an effective coupling constant up to the Planck distance, which then also become frozen. Therefore quantum gravitation not only is finite, but also there works the perturbation theory and the sums of loops are convergent.

At more realistic and detail considerations the conclusions of the above presented simple model estimations will be mainly remain the same since the time dilation is a fundamental property of gravitation and it is inevitable at any exact theory of quantum gravity effects. 


\section{Conclusion}

Thus, at the correcting of erroneous assumptions of BHP, incompatible with $\mathrm{GR}$, the horizon and singularities disappear, and instead of the collapse and the black holes GR predicts in fact the new phase state of matter - the gravitationally frozen states in the superdense and supermassive objects.

The surface radii of gravitationally frozen objects always exceed their gravitational radius, and their center is always more frozen than their surface. The gravitationally frozen matter is cold in world time and has low coordinate temperature, but its proper temperature and pressure are very high.

As the result, in astrophysics GR predicts a hierarchy of superdense stars of various matter content (baryons, quarks, bosons), and also supermassive objects which are inhomogeneously frozen - quasars and AGNs, at which the central parts are superdense and are highly frozen, while the surface layers can have usual densities, although with large time dilation coefficients.

In cosmology there are strong reasons to consider the Universe as a closed 3D sphere embedded into 4D space, the expansion of which occurs along fourth spatial dimension. Then in GR the cosmological singularity at initial moment does not arise, Big-Bang is refreezing of the Universe, and age of the Universe in the time scales of our epoch should be much more than the proper age of the matter.

In particle physics GR leads to a suitable result that at the Planck distances the quantum fluctuations freeze due to the external gravitational field of the loop and frequencies of quanta in loops always are less than the Planck frequency. Thus, in GR there is a natural regularization of any loop contributions and, therefore, the theories of quantum fields, including the quantum gravity, are ultraviolet finite ones.

\section{References}

1. Einstein A. (1916) Ann. d. Phys., 49, 769.

2. Einstein A. (1939) Ann. Math., 40, p. 922.

3. Schwarzschild K. (1916) Sitz. Preuss. Akad. Wiss., p. 189.

4. Oppenheimer J.R., Snyder H. (1939) Phys. Rev. 56, p. 455.

5. Misner C.W., Thorne K.S., Wheeler J.A. Gravitation. Freeman, 1973.

6. Penrose R. (1968) Structure of Space-Time. Batt.Ren., Benjamin, N. Y.

7. Hawking S.W., Ellis G. (1973) Large Scale Struct. Space-Time. C.U.P.

8. Thorne K.S. et al (1986) Black Holes: The Membrane Paradigm. Y.U.P.

9. Frolov V.P., Novikov I.D. (1998) Black Hole Physics. Kluwer.

10. Narayan R. (2005) New Journal of Phys., 7, p.199.

11. Feynman R., et al (1995) Feynman Lect. on Grav., Add.-Wes.P.C.

12. Mitra A. (2005) arXiv:astro-ph/0608178.

13. Zakir Z. (2000) arXiv:physics/000709.

14. Will C.M. (2006) Living Rev. Rel. 9, 3.

15. Haefele J., Keating R. (1972) Science, 117, pp.168, 170.

16. Alley C et al. (1976) Exp. Grav., Proc. Conf. at Pavia, N.Y., Acad. Press.

17. Okun L.B., Selivanov K.G., Telegdi V.L. (2000) Amer. J. Phys., 68, 115; arXiv:physics/9907017.

18. Zakir Z. (2006) Theor. Phys., Astroph. \& Cosmol., 1, 1, p. 11; doi: 10.9751/TPAC.2091-002; 1, 2, p. 28; doi: 10.9751/TPAC.2098-003 